\title{
ALGUNOS ASPECTOS DEL SECTOR DEL COMERCIO EN EL AMBITO LOCAL
}

$381: 311(46)(-2)$

por

Ignacio Ballester Ros

SUMARIO: I. INTRODUCCION.-II. LOS PRINCIPALES CENTROS COMERCIALES ESPAÑOLES.-III. EL INCREMENTO EN LOS ULTIMOS AÑOS: 1. Del CONSUMO ENERGÉtico. 2. De la CAPACIDAD DE COMPRA POR HABITANTE. 3. DE LAS LICENCIAS COMERCIALES.-IV. EL INDICE DE VIVACIDAD DE LAS PROVINCIAS.-V. LA EVOLUCION DEL CONJUNTO DE MUNICIPIOS ENTRE 1.000 Y $3.000 \mathrm{HA}$ BITANTES.-VI. ANALISIS ESPECIAL DE VALENCIA Y SU CONTORNO.

\section{INTRODUCCION}

El Banco Español de Crédito acaba de publicar la duodécima edición del Anuario del Mercado Español, obra ya clásica, caracterizada por estudios de gran calidad, unos de carácter periódico y otros especiales, que son verdaderas primicias sobre aspectos diversos de nuestra estructura y coyuntura económica, que constituye un "eficaz instrumento para el conocimiento de la dinámica y de la compleja realidad del mercado nacional».

En trabajos anteriores, publicados en esta REvisTa, se ha expuesto, con detalle, el contenido y sistemática del Anuario. Prosigue la elaboración de índices e indicadores, que forman series cronológicas de indudable importancia, y en los que radica una de las principales utilidades de la obra, y presenta, además, como todos los años, estudios específicos de primera mano, fruto de en- 
cuestas realizadas por el equipo de estadísticos y economistas que elaboran la publicación. En esta edición se insertan dos trabajos especiales: en el primero se estudian e identifican los 500 centros comerciales más importantes de España, y en el segundo se presentan los resultados de una encuesta muestral dirigida a los hogares que integran el núcleo de Valencia y su contorno, que configura una conurbación de características bien definidas, en orden al grado de consumo, hábitos de compra y equipamiento de los hogares, que permiten un conocimiento adecuado de este mercado, una de las Areas metropolitanas más importantes, que completa el estudio, hecho con anterioridad, de Madrid y Barcelona.

Dada la amplitud informativa y. la riqueza de contenido del Anuario, hemos de seleccionar forzosamente algunos temas como base del comentario de esta Crónica estadística, centrándolo, de una parte, en la contemplación de la evolución de las Provincias, en el orden comercial, y de otra, en el análisis de los Municipios, tendencia muy marcada por los numerosos trabajos orientados al estudio de los problemas de la Vida local.

\section{LOS PRINCIPALES CENTROS COMERCIALES ESPAÑOLES}

En 1963, el Consejo Superior de Cámaras de Comercio, Industria y Navegación de España publicó el Atlas Comercial de España, elaborado por el mismo equipo que hoy tiene a su cargo este Anuario, en el que quedaron perfectamente delimitadas las áreas y subáreas comerciales de España.

Ultimamente se ha realizado una nueva investigación basada en el Municipio y tendente a identificar las principales plazas comerciales, en función de tres variables: su población, su distribución en superficie y las posibilidades de compra de los habitantes que la integran, sistema que perfecciona y mejora los cálculos basados en la cuota de mercado. Así se han obtenido coeficientes que valoran la importancia de cada centro comercial, en función de la población agrupada en núcleos de 2.000 y más habitantes, la población dispersa y ubicada en núcleos de menos de 2.000 habitantes y el nivel medio de renta del Municipio.

De esta forma se han fijado los 500 Municipios de mayor importancia comercial, cuya población es de 22,3 millones de habi- 
tantes, lo que equivale al 65,8 por 100 de la población total. Se han clasificado en 20 grupos de coeficientes, que hemos reducido, para mayor concisión, a los seis siguientes:

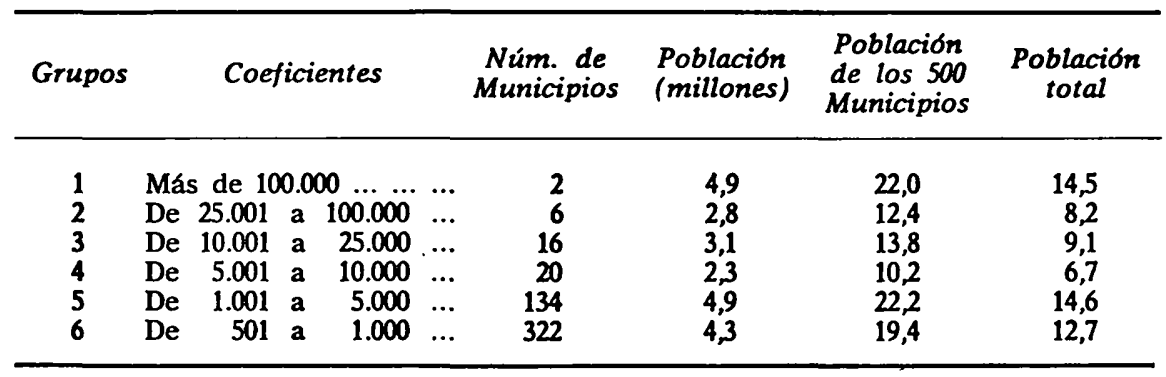

Entre estos 500 Municipios figuran todos los mayores de 20.000 habitantes, el 76 por 100 de los de población comprendida entre 10.001 y 20.000 y la sexta parte de los de 501 y 10.000 habitantes.

Destacan sobremanera Madrid y Barcelona, con la sexta parte de la población total, y los seis del segundo grupo: Valencia, Sevilla, Bilbao, Zaragoza, Málaga y Las Palmas. En el Anuario se inserta un mapa en el que se presenta la ubicación de cada uno de estos 500 Municipios, y su distribución por Provincias es como sigue:

\begin{tabular}{|c|c|c|}
\hline Provincias & $\begin{array}{c}\text { Núm. de } \\
\text { Municipios }\end{array}$ & $\begin{array}{l}\% \text { de la } \\
\text { población } \\
\text { provincial }\end{array}$ \\
\hline 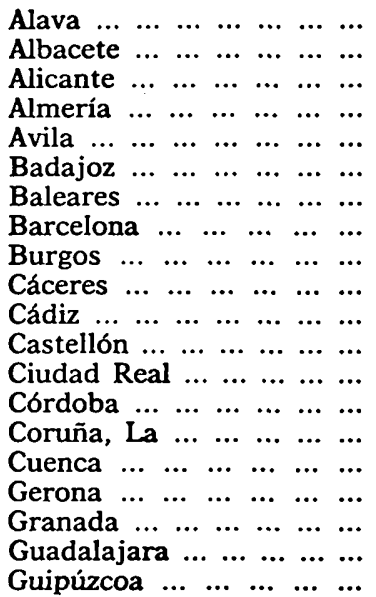 & $\begin{array}{r}2 \\
5 \\
24 \\
6 \\
2 \\
19 \\
15 \\
56 \\
3 \\
3 \\
16 \\
9 \\
12 \\
12 \\
11 \\
1 \\
15 \\
6 \\
1 \\
19\end{array}$ & $\begin{array}{l}74,6 \\
49,0 \\
76,7 \\
50,0 \\
18,0 \\
37,6 \\
72,7 \\
89,4 \\
48,1 \\
20,4 \\
67,5 \\
61,8 \\
52,0 \\
61,6 \\
47,7 \\
14,0 \\
50,1 \\
40,5 \\
21,6 \\
76,2\end{array}$ \\
\hline
\end{tabular}




\begin{tabular}{|c|c|c|}
\hline Provincias & $\begin{array}{l}\text { Núm. de } \\
\text { Municipios }\end{array}$ & $\begin{array}{l}\% \text { de la } \\
\text { población } \\
\text { provincial }\end{array}$ \\
\hline $\begin{array}{lllllll}\text { Huelva } & \ldots & \ldots & \ldots & \ldots & \ldots & \ldots\end{array}$ & 3 & 31,2 \\
\hline $\begin{array}{lllllll}\text { Huesca } & \ldots & \ldots & \ldots & \ldots & \ldots & \ldots\end{array}$ & 7 & 43,7 \\
\hline $\begin{array}{llllllll}\text { Jaén } & \ldots & \ldots & \ldots & \ldots & \ldots & \ldots & \ldots\end{array}$ & 11 & 46,1 \\
\hline $\begin{array}{llllllll}\text { León } & \ldots & \ldots & \ldots & \ldots & \ldots & \ldots & \ldots\end{array}$ & 7 & 31,3 \\
\hline $\begin{array}{lllllllll}\text { Lérida } & \ldots & \ldots & \ldots & \ldots & \ldots & \ldots & \ldots\end{array}$ & 6 & 38,5 \\
\hline $\begin{array}{lllllll}\text { Logroño } & \ldots & \ldots & \ldots & \ldots & \ldots & \ldots\end{array}$ & 7 & 58,7 \\
\hline $\begin{array}{llllllll}\text { Lugo } & \ldots & \ldots & \ldots & \ldots & \ldots & \ldots & \ldots\end{array}$ & 5 & 30,3 \\
\hline $\begin{array}{lllllll}\text { Madrid } & \ldots & \ldots & \ldots & \ldots & \ldots & \ldots\end{array}$ & 17 & 94,0 \\
\hline $\begin{array}{lllllll}\text { Málaga } & \ldots & \ldots & \ldots & \ldots & \ldots & \ldots\end{array}$ & 12 & 72,5 \\
\hline $\begin{array}{lllllll}\text { Murcia } & \ldots & \ldots & \ldots & \ldots & \ldots & \ldots\end{array}$ & 21 & 87,0 \\
\hline $\begin{array}{lllllll}\text { Navarra } & \ldots & \ldots & \ldots & \ldots & \ldots & \ldots\end{array}$ & 4 & 40,3 \\
\hline $\begin{array}{llllllll}\text { Orense } & \ldots & \ldots & \ldots & \ldots & \ldots & \ldots & \ldots\end{array}$ & 1 & 17,7 \\
\hline $\begin{array}{llllllll}\text { Oviedo } & \ldots & \ldots & \ldots & \ldots & \ldots & \ldots & \ldots\end{array}$ & 19 & 76,6 \\
\hline $\begin{array}{lllllll}\text { Palencia } & \ldots & \ldots & \ldots & \ldots & \ldots & \ldots\end{array}$ & 2 & 33,9 \\
\hline $\begin{array}{lllll}\text { Palmas }(\text { Las) } & \ldots & \ldots & \ldots & \ldots\end{array}$ & 9 & 79,3 \\
\hline $\begin{array}{llllll}\text { Pontevedra } & \ldots & \ldots & \ldots & \ldots & \ldots\end{array}$ & 9 & 52,4 \\
\hline $\begin{array}{llllll}\text { Salamanca } & \ldots & \ldots & \ldots & \ldots & \ldots\end{array}$ & 3 & 42,0 \\
\hline Santa Cruz de Tenerife .... & 14 & 74,6 \\
\hline $\begin{array}{llllll}\text { Santander } & \ldots & \ldots & \ldots & \ldots & \ldots\end{array}$ & 8 & 56,0 \\
\hline $\begin{array}{lllllll}\text { Segovia } & \ldots & \ldots & \ldots & \ldots & \ldots & \ldots\end{array}$ & 3 & 33,0 \\
\hline $\begin{array}{lllllll}\text { Sevilla } & \ldots & \ldots & \ldots & \ldots & \ldots & \ldots\end{array}$ & 20 & 73,3 \\
\hline $\begin{array}{cccccccc}\text { Soria } & \ldots & \ldots & \ldots & \ldots & \ldots & \ldots & \ldots\end{array}$ & 1 & 21,8 \\
\hline $\begin{array}{llllll}\text { Tarragona } & \ldots & \ldots & \ldots & \ldots & \ldots\end{array}$ & 9 & 56,9 \\
\hline 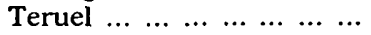 & 2 & 19,1 \\
\hline $\begin{array}{lllllll}\text { Toledo } & \ldots & \ldots & \ldots & \ldots & \ldots & \ldots\end{array}$ & 5 & 24,5 \\
\hline $\begin{array}{llllllll}\text { Valencia } & \ldots & \ldots & \ldots & \ldots & \ldots & \ldots\end{array}$ & 41 & 76,3 \\
\hline $\begin{array}{lllllll}\text { Valladolid } & \ldots & \ldots & \ldots & \ldots & \ldots\end{array}$ & 3 & 62,5 \\
\hline $\begin{array}{lllllll}\text { Vizcaya } & \ldots & \ldots & \ldots & \ldots & \ldots & \ldots\end{array}$ & 17 & 79,8 \\
\hline $\begin{array}{lllllll}\text {ZZamora} & \ldots & \ldots & \ldots & \ldots & \ldots & \ldots\end{array}$ & 3 & 28,1 \\
\hline $\begin{array}{lllllll}Z & Z\end{array}$ & 4 & 68,8 \\
\hline
\end{tabular}

Los centros comerciales de mayor importancia se sitúan en las 18 Provincias con porcentaje de población, por ellos atendida, superior al nacional, de 65,8 , y que configuran estas zonas:

Norte: Oviedo, Vizcaya, Guipúzcoa, Alava.

Centro-Norte: Madrid y Valladolid, en Castilla, y Zaragoza, en el Valle del Ebro.

Litoral mediterráneo: Barcelona, Baleares, Castellón, Valencia, Alicante y Murcia.

Sur: Cádiz, Sevilla, Córdoba y Málaga.

Canarias: con sus dos Provincias.

Si bien destacan, por sus concentraciones urbanas, Madrid, Barcelona y Murcia.

Por contraste, con más del 75 por 100 de su población carente de centros comerciales importantes se encuentran: Orense y Cáceres; Avila, Soria, Guadalajara, Cuenca, Toledo y Teruel. 


\section{EL INCREMENTO EN LOS ULTIMOS AÑOS}

En el último decenio, debido al desarrollo general de nuestra economía, se han producido incrementos sustanciales en el consumo de energía y de bienes consuntivos y de capital, aunque con algunos cambios coyunturales en el consumo de algunos productos, y ha aumentado el número de establecimientos comerciales. Las series cronológicas, publicadas en los Anuarios del Mercado Español y sintetizadas en el último, nos permiten seguir de cerca esta evolución a nivel provincial.

\section{Del CONSUmo ENERGÉtico}

No todos los combustibles registran aumentos análogos en su consumo. En el conjunto nacional, los índices de incremento, base: $1963=100$, presentan para 1974 los siguientes valores:

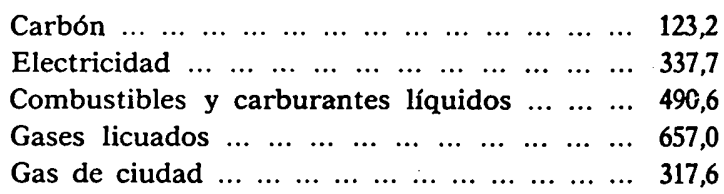

Los gases licuados, para usos domésticos e industriales, han registrado una notable expansión que, en cuanto al uso doméstico se refiere, se ha extendido a toda el área nacional; le siguen en orden de importancia del aumento de su consumo los carburantes y combustibles líquidos, debido al desarrollo del parque automovilístico nacional y a la generalización del uso de subproductos petroliferos en la industria, el transporte ferroviario y en la calefacción de viviendas, todo ello en detrimento del consumo del carbón, que ha caído en desuso en varios sectores, $y$, finalmente, la electricidad, cuyo consumo se ha incrementado en la industria y en los hogares, y que, en cuanto a estos últimos se refiere, es un indicador del aumento del nivel de vida, pues ha hecho posible un alto grado de utilización de aparatos electrodomésticos.

El detalle provincial de los citados índices de incremento es éste: 


\begin{tabular}{|c|c|c|c|c|c|}
\hline Provincias & Carbon & $\begin{array}{l}\text { Electri- } \\
\text { cidad }\end{array}$ & $\begin{array}{l}\text { Combusti- } \\
\text { bles y car- } \\
\text { burantes } \\
\text { liquidos }\end{array}$ & $\begin{array}{c}\text { Gases } \\
\text { licuados }\end{array}$ & $\begin{array}{c}\text { Gas } \\
\text { ciudad }\end{array}$ \\
\hline $\begin{array}{lllllll}\text { Alava } & \ldots & \ldots & \ldots & \ldots & \ldots\end{array}$ & 34,5 & 605,1 & 709,4 & $1.012,0$ & \\
\hline Albacete $\ldots \ldots \ldots \ldots$ & 87,3 & 400,9 & 403,7 & 714,3 & \\
\hline $\begin{array}{lllll}\text { Alicante } & \ldots & \ldots & \ldots & \ldots \\
\end{array}$ & 14,0 & 401,4 & 154,0 & 694,6 & \\
\hline $\begin{array}{lllll} & \ldots l & & & \\
& & \ldots & \ldots & \ldots\end{array}$ & 3,0 & 334,8 & 447,7 & $1.330,0$ & \\
\hline 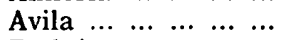 & 43,1 & 274,3 & 651,7 & $1: 566,7$ & \\
\hline $\begin{array}{lllll}\text { Badajoz } & \ldots & \ldots & \ldots & \ldots \\
\end{array}$ & 2,8 & 269,2 & 268,4 & 1.236 .4 & \\
\hline Baleares $\ldots \ldots \ldots \ldots$ & 54,2 & 548,2 & 526,4 & 948,6 & 471,8 \\
\hline $\begin{array}{llll}\text { Barcelona } & \ldots & \ldots & \ldots\end{array}$ & 37,6 & 319,2 & 406,7 & 924,9 & 281,4 \\
\hline $\begin{array}{llllll}\text { Burgos } & \ldots & \ldots & \ldots & \ldots\end{array}$ & 40,3 & 355,6 & 756,2 & $1.227,3$ & \\
\hline $\begin{array}{lllll}\text { Cáceres } & \ldots & \ldots & \ldots & \ldots\end{array}$ & - & 244,3 & 350,9 & $1.110,0$ & \\
\hline $\begin{array}{llllll}\text { Cádiz } & \ldots & \ldots & \ldots & \ldots & \ldots\end{array}$ & 5,8 & 433,6 & 624,6 & 815,4 & 580,0 \\
\hline $\begin{array}{llll}\text { Castellón } & \ldots & \ldots & \ldots\end{array}$ & 1,4 & 513,2 & $2.096,6$ & $1.564,3$ & \\
\hline Ciudad Real ... ... & 43,4 & 295,4 & 434,1 & $1.450,0$ & \\
\hline Córdoba $\ldots \ldots \ldots$ & 248,4 & 234,6 & 418,5 & 557,5 & \\
\hline Coruña, La $\ldots . . . . .$. & 169,5 & 422,2 & 402,0 & 224,0 & \\
\hline $\begin{array}{ccccc}\text { Cuenca } & \ldots & \ldots & \ldots & \ldots\end{array}$ & 584,6 & 541,4 & 410,7 & $6.650,0$ & \\
\hline $\begin{array}{ccccc}\text { Gerona } & \ldots & \ldots & \ldots & \ldots\end{array}$ & 6,1 & 388,4 & 487,7 & 597,5 & 400,0 \\
\hline $\begin{array}{lllll}\text { Granada } & \ldots & \ldots & \ldots & \ldots\end{array}$ & 9,6 & 414,3 & 447,6 & 381,3 & \\
\hline $\begin{array}{ccc}\text { Guadalajara } & \ldots & \ldots\end{array}$ & 57,8 & 667,5 & 636,9 & $12.225,0$ & \\
\hline $\begin{array}{lllll}\text { Guipúzcoa } & \ldots & \ldots & \ldots\end{array}$ & 103,8 & 302,3 & 435,0 & 221,8 & 265,9 \\
\hline $\begin{array}{llllll}\text { Huelva } & \ldots & \ldots & \ldots & \ldots\end{array}$ & 41,0 & 609,6 & 579,4 & 888,9 & \\
\hline $\begin{array}{lllll}\text { Huesca } & \ldots & \ldots & \ldots & \ldots\end{array}$ & 70,1 & 276,7 & 427,0 & $2.075,0$ & \\
\hline 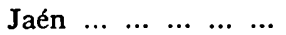 & 39,9 & 296,3 & 319,5 & $1.096,2$ & \\
\hline León $\ldots \ldots \ldots$ & 333,3 & 206,7 & 749,6 & $2.014,3$ & \\
\hline $\begin{array}{llllll}\text { Lérida } & \ldots & \ldots & \ldots & \ldots\end{array}$ & 7,8 & 344,6 & 357,8 & 794,1 & 780,0 \\
\hline $\begin{array}{llllll}\text { Logroño } & \ldots & \ldots & \ldots & \ldots & \ldots\end{array}$ & 6,7 & 376,2 & 648,9 & 752,9 & \\
\hline $\begin{array}{lllllll}\text { Lugo } & \ldots & \ldots & \ldots & \ldots & \ldots\end{array}$ & 270,8 & 300,2 & 652,2 & $1.875,0$ & \\
\hline $\begin{array}{lllll}\text { Madrid } & \ldots & \ldots & \ldots & \ldots\end{array}$ & 30,7 & 396,3 & 499,5 & 208,1 & 365,4 \\
\hline $\begin{array}{lllll}\text { Málaga } & \ldots & \ldots & \ldots & \ldots\end{array}$ & 5,3 & 565,9 & 468,1 & 804,9 & 612,5 \\
\hline $\begin{array}{lllll}\text { Murcia } & \ldots & \ldots & \ldots & \ldots\end{array}$ & 44,2 & 359,4 & 403,8 & 912,5 & 328,6 \\
\hline $\begin{array}{ccccc}\text { Navarra } & \ldots & \ldots & \ldots & \ldots\end{array}$ & 10,9 & 420,4 & 618,8 & 645,8 & \\
\hline $\begin{array}{lllll}\text { Orense } & \ldots & \ldots & \ldots & \ldots\end{array}$ & 104,1 & 211,7 & 576,8 & $2.100,0$ & \\
\hline $\begin{array}{lllll}\text { Oviedo } & \ldots & \ldots & \ldots & \ldots\end{array}$ & 181,6 & 292,3 & $1.154,4$ & $2.293,3$ & 266,7 \\
\hline Palencia $. . . \quad \ldots \ldots . .$. & 253,7 & 265,0 & 789,5 & $1.450,0$ & \\
\hline Palmas, Las ... ... ... & - & 719,3 & - & - & \\
\hline Pontevedra $\ldots . . . . .$. & 24,9 & 403,6 & 201,5 & 1.631 .2 & \\
\hline $\begin{array}{cccc}\text { Salamanca } & \ldots & \ldots & \ldots\end{array}$ & 51,5 & 255,0 & 471,3 & 588,2 & $\cdot$ \\
\hline Sta. Cruz Tenerife. & - & 303,6 & - & - & 6,6 \\
\hline $\begin{array}{llll}\text { Santander } & \ldots & \ldots & \ldots\end{array}$ & 52,5 & 260,4 & 555,2 & $1.286,7$ & 230,8 \\
\hline $\begin{array}{lllll}\text { Segovia } & \ldots & \ldots & \ldots & \ldots\end{array}$ & 17,5 & 391,5 & 368,8 & $1.375,0$ & \\
\hline $\begin{array}{lllll}\text { Sevilla } & \ldots & \ldots & \ldots & \ldots\end{array}$ & 8,9 & 340,8 & 271,6 & 832,1 & 452,6 \\
\hline $\begin{array}{lllllll}\text { Soria } & \ldots & \ldots & \ldots & \ldots & \ldots\end{array}$ & 16,7 & 552,3 & 469,6 & 933,3 & \\
\hline $\begin{array}{llll}\text { Tarragona } & \ldots & \ldots & \ldots\end{array}$ & 45,8 & 568,8 & 251,6 & $1.104,8$ & 475,0 \\
\hline $\begin{array}{llllll}\text { Teruel } & \ldots & \ldots & \ldots & \ldots\end{array}$ & 336,2 & 252,9 & 470,4 & $1.100,0$ & \\
\hline $\begin{array}{lllll}\text { Toledo } & \ldots & \ldots & \ldots & \ldots\end{array}$ & 1,8 & 496,4 & $1.402,0$ & $1.084,6$ & \\
\hline $\begin{array}{lllll}\text { Valencia } & \ldots & \ldots & \ldots & \ldots\end{array}$ & 97,7 & 361,3 & 346,8 & 985,1 & 193,9 \\
\hline $\begin{array}{lllll}\text { Valladolid } & \ldots & \ldots & \ldots\end{array}$ & 25,4 & 240,6 & 554,0 & $1.837,5$ & 720,0 \\
\hline $\begin{array}{lllll}\text { Vizcaya } & \ldots & \ldots & \ldots & \ldots\end{array}$ & 108,1 & 262,5 & 678,4 & 959,7 & 150,0 \\
\hline $\begin{array}{lllll}\text { Zamora } & \ldots & \ldots & \ldots & \ldots\end{array}$ & 38,9 & 277,6 & 468,9 & $1.040,0$ & \\
\hline Zaragoza $\ldots \ldots \ldots \ldots$ & 89,5 & 355,9 & 474,3 & 844,1 & 329,6 \\
\hline
\end{tabular}


La evolución del consumo energético en el período 1963-1974 es muy significativo, tanto cualitativa como cuantitativamente. El consumo de carbón, debido a su sustición por otras fuentes de energía, ha registrado, en el conjunto nacional, un incremento muy bajo, del 23 por 100, que sólo superan siete Provincias, clásicamente mineras de estos combustibles o lindantes con ellas, como $\mathrm{La} \mathrm{Co}$ ruña, Lugo, León y Palencia; Teruel y Cuenca, y Córdoba.

El consumo de gas de ciudad y de electricidad se ha más que duplicado en los últimos doce años. Superan el incremento nacional 11 de las 18 capitales que disponen de fábricas de gas y destacan por sus tasas más altas, Lérida, Valladolid y Málaga. El consumo de electricidad está más generalizado; 28 son las Provincias con aumentos que rebasan el promedio nacional, que se hallan distribuidas en todas las Regiones, dándose la circunstancia de que las tasas más elevadas corresponden a las de consumo más bajo en 1963.

En cuanto a carburantes y combustibles liquidos, su consumo se ha multiplicado por cuatro en el mismo período, si bien el área de altas tasas, superiores a la media nacional, se limita a 20 Provincias, distribuidas igualmente en todas las Regiones, repitiéndose la circunstancia que algunas de ellas responden a consumos mínimos en el año inicial del período. Los mayores incrementos de consumo son los de gases licuados, 5,6 veces superiores a los de 1963 en el conjunto nacional; de tal forma se ha generalizado, que hasta 41 Provincias tienen tasas superiores a la nacional.

Los consumos de estos elementos energéticos para 1974 -para carbón y electricidad, 1973-, expresados en miles de unidades TEC, permiten conocer la importancia de las Provincias en total consumo energético, teniendo en cuenta que el consumo medio nacional es de $2.186,1$ miles de TEC. El detalle provincial es como sigue:

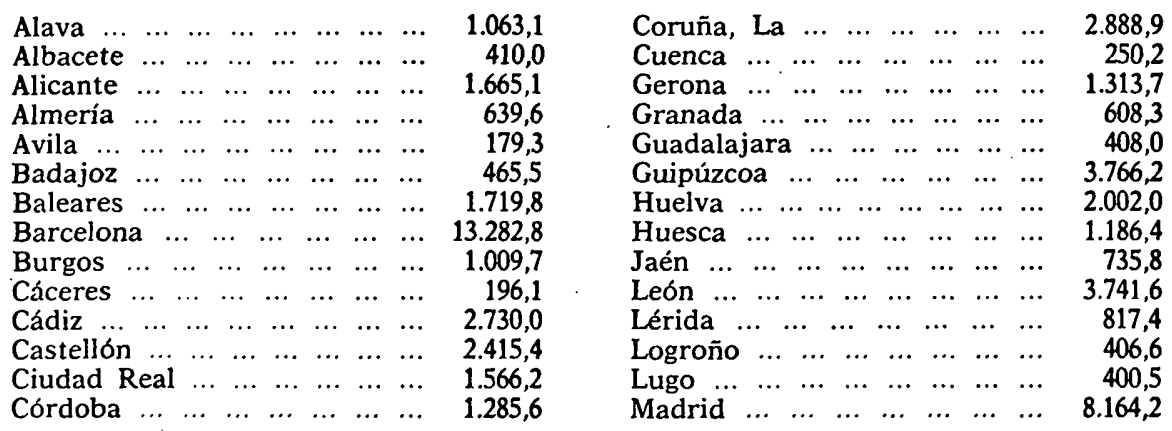




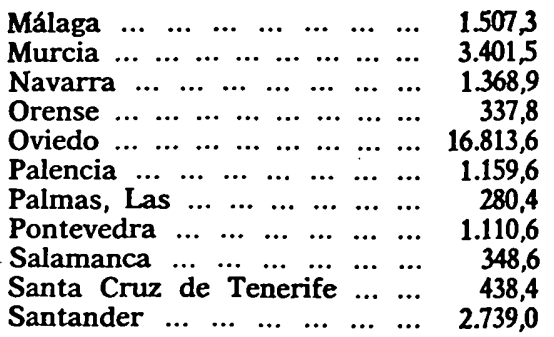

\begin{tabular}{|c|c|}
\hline 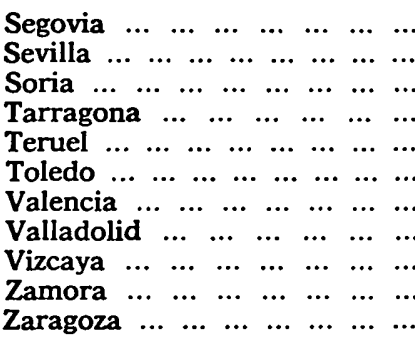 & $\begin{array}{r}211,8 \\
2.229,9 \\
182,1 \\
1.467,5 \\
676,9 \\
2.253,3 \\
4.517,6 \\
1.366,7 \\
9.097,1 \\
244,6 \\
2.234,7\end{array}$ \\
\hline
\end{tabular}

Superan la media nacional los consumos energéticos de 15 Provincias, que configuran estas zonas:

Norte.-Con La Coruña, Oviedo -que registra el máximo provincial-, León, Santander, Vizcaya - la tercera- y Guipúzcoa, predominantemente industriales.

Litoral mediterráneo.-Con Barcelona - la segunda por el volumen de su consumo-, Castellón, Valencia y Murcia.

Y, además, Zaragoza; Madrid - la cuarta-, y Toledo; Cádiz y Sevilla.

La Galicia interior, el Valle del Duero, la Submeseta Sur y Canarias presentan las tasas mínimas, inferiores al medio millón de TEC anuales.

\section{DE LA CAPACIDAD DE COMPRA POR HABITANTE}

Los índice de capacidad de compra provincial tratan de determinar la cantidad de bienes y de servicios que, teóricamente y en igualdad de condiciones, pueden absorber las Provincias. Se elaboran tres índices: el primero mide la capacidad de compra de "productos de uso y consumo común, caracterizado por su bajo coste unitario y la posibilidad de adquirirlo normalmente en cualquier tipo de núcleos, aun en aquellos escasamente dotados de equipo comercial»; el segundo se refiere a los «artículos de tipo medio que, aun no siendo fuertemente especializados, su uso o consumo ya no está en función del volumen de población», y el tercero se aplica a "productos de uso y consumo muy especializado".

Teniendo en cuenta los índices citados, se han obtenido los índices cronológicos provinciales de capacidad de compra por habitante, período 1965-1974, base 1965 igual a la unidad, que para 1974 son los siguientes: 


\begin{tabular}{|c|c|c|c|}
\hline Provincias & $10^{\circ}$ & $20^{\circ}$ & $3 .^{\circ}$ \\
\hline 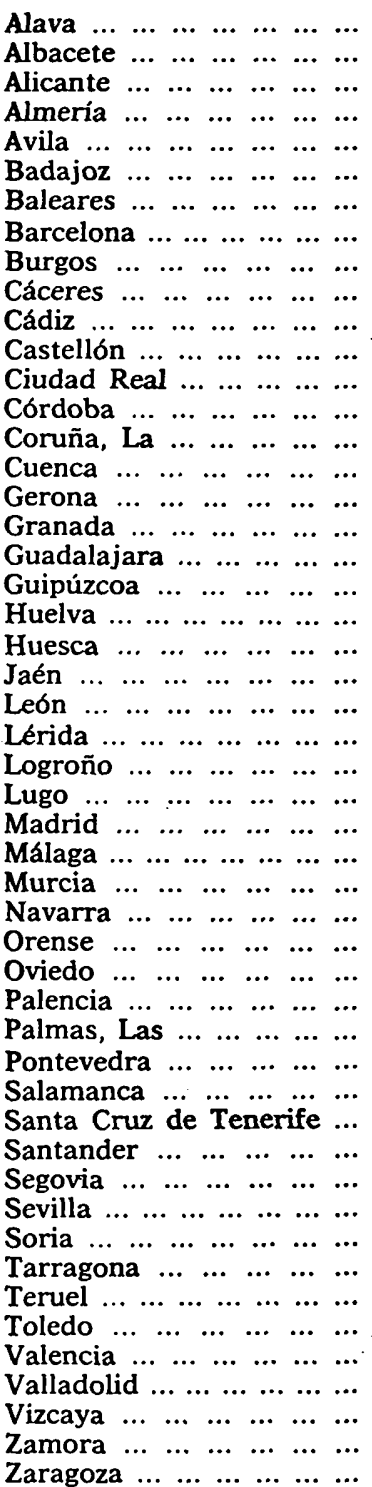 & $\begin{array}{l}1,73 \\
2,30 \\
2,02 \\
2,11 \\
2,17 \\
1,95 \\
1,65 \\
1,58 \\
2,11 \\
2,02 \\
2,20 \\
1,87 \\
2,15 \\
1,97 \\
2,21 \\
2,21 \\
1,16 \\
1,95 \\
2,61 \\
1,40 \\
2,31 \\
1,51 \\
1,95 \\
2,27 \\
1,80 \\
2,04 \\
2,28 \\
1,55 \\
2,00 \\
1,97 \\
1,78 \\
2,23 \\
1,85 \\
2,21 \\
1,74 \\
2,19 \\
2,10 \\
1,95 \\
1,64 \\
2,46 \\
2,02 \\
2,21 \\
1,80 \\
2,10 \\
2,24 \\
1,91 \\
1,95 \\
1,66 \\
2,15 \\
1,89\end{array}$ & $\begin{array}{l}1,89 \\
2,79 \\
2,39 \\
2,63 \\
3,04 \\
2,37 \\
1,94 \\
1,77 \\
2,54 \\
2,29 \\
2,59 \\
2,25 \\
2,65 \\
2,20 \\
2,54 \\
3,35 \\
1,64 \\
2,35 \\
3,62 \\
1,64 \\
3,05 \\
1,72 \\
2,20 \\
2,39 \\
2,13 \\
2,45 \\
2,78 \\
1,80 \\
2,36 \\
2,35 \\
1,95 \\
2,53 \\
1,99 \\
2,51 \\
1,86 \\
2,55 \\
2,50 \\
2,08 \\
1,84 \\
3,36 \\
2,35 \\
2,75 \\
2,04 \\
2,70 \\
2,73 \\
2,16 \\
2,27 \\
1,89 \\
2,60 \\
2,18\end{array}$ & $\begin{array}{l}2,23 \\
3,43 \\
2,88 \\
3,39 \\
3,93 \\
2,99 \\
2,22 \\
2,02 \\
3,08 \\
3,06 \\
3,16 \\
4,71 \\
3,41 \\
2,85 \\
3,34 \\
3,85 \\
1,97 \\
2,92 \\
4,45 \\
1,99 \\
3,99 \\
2,34 \\
2,68 \\
3,07 \\
2,57 \\
3,03 \\
3,68 \\
2,06 \\
2,94 \\
2,86 \\
2,14 \\
3,39 \\
2,49 \\
3,22 \\
2,10 \\
3,16 \\
3,04 \\
2,34 \\
2,23 \\
4,33 \\
.2,88 \\
3,61 \\
2,44 \\
3,47 \\
3,68 \\
2,54 \\
2,83 \\
2,22 \\
3,35 \\
2,57\end{array}$ \\
\hline
\end{tabular}


Los índices cronológicos nacionales de capacidad de compra por habitante para el año de referencia son:

\section{Primero: 1,89}

Segundo: 2,20

Tercero: 2,62

de los que se deduce que si en el período que consideramos es evidente que se ha incrementado la capacidad de compra de productos de uso y consumo común, queda igualmente patente que aún ha sido mayor el aumento de la capacidad de compra de artículos de mediana especialización y, sobre todo, de los artículos muy especializados.

La distribución provincial corrobora la afirmación anterior en toda su amplitud y, dentro de esta tónica, ofrece una marcada homogeneidad al considerar las Provincias - 35, 35 y 33, respectivamente- que presentan incrementos superiores a los nacionales en los tres índices. Una vez más se advierte que los índices más elevados corresponden, por lo general, a Provincias de economía predominantemente agraria, con bajos consumos en 1965, y que han perdido habitantes en el último decenio.

\section{DE LAS LICENCIAS COMERCIALES}

Es clásica la información contenida en el Anuario sobre la evolución de las licencias comerciales de mayoristas y minoristas. Solamente hemos tomado el dato relativo al porcentaje de incremento del número de licencias, de ambas clases, en 1975 sobre las existentes en 1966.

Estos valores son indicativos para conocer el desarrollo del comercio, si bien hay que tener en cuenta el proceso de concentración de empresas y el establecimiento de cadenas comerciales de supermercados y autoservicios operado en los últimos años.

En el conjunto nacional dicho aumento ha sido del 24,1 por 100 de mayoristas y del 21,4 por 100 de minoristas. El detalle por Provincias ha sido éste: 


\begin{tabular}{|c|c|c|c|c|}
\hline Provincias & & Mayoristas & & Minoristas \\
\hline 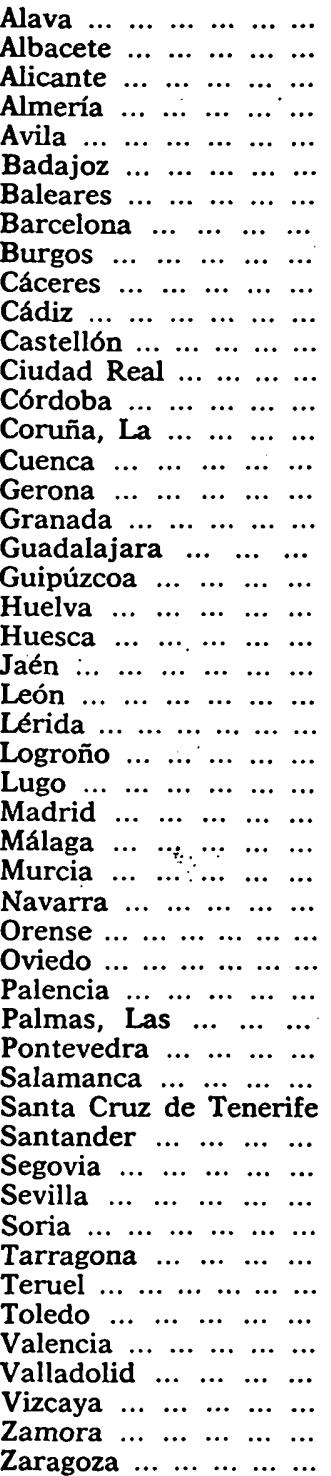 & 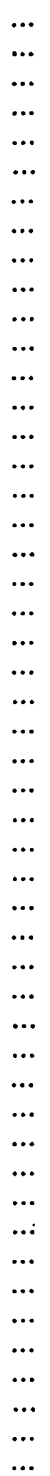 & 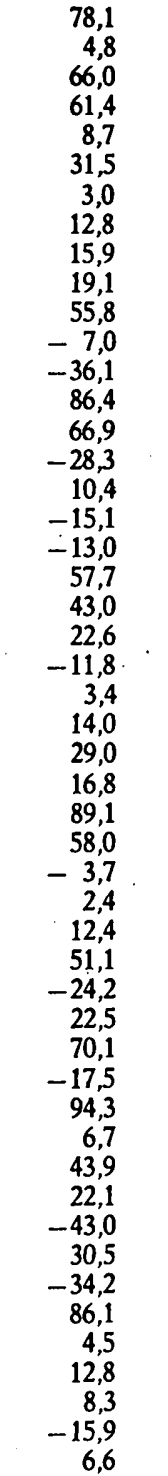 & $\cdot$ & 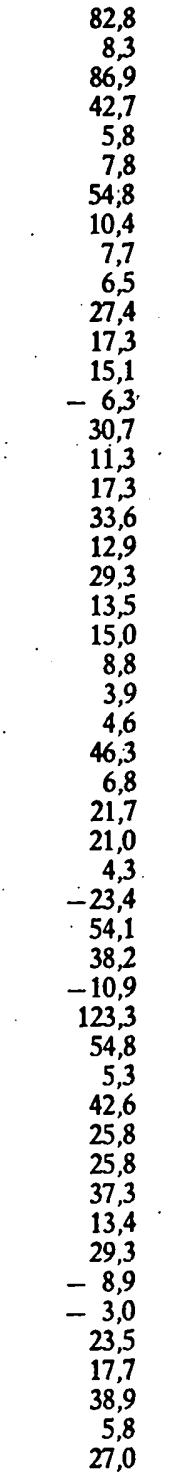 \\
\hline
\end{tabular}

A pesar de que, en el conjunto del país, el incremento del comercio mayorista ha sido superior al del minorista, el primero presenta incrementos superiores al segundo en sólo 23 Provincias, al 
propio tiempo que registra bajas apreciables en otras 12, preferentemente agrarias, de ambas Mesetas, Andalucía y Murcia. Palencia y Teruel ofrecen valores negativos en ambas clases de establecimientos.

El incremento de establecimientos mayoristas ha sido superior al nacional en 18 Provincias, y el de minoristas, en 22. Es de destacar el desarrollo paralelo de ambos tipos de establecimientos comerciales en 13 Provincias, que, geográficamente, se distribuyen en esta forma:

Norte.-La Coruña y Pontevedra, Oviedo, Guipúzcoa, Alava y Logroño.

Centro.--Madrid y Segovia.

Sur.-Almería y Cádiz, los dos puntos extremos de Andalucía, y Santa Cruz de Tenerife.

Litoral mediterráneo.-Tarragona y Alicante.

\section{EL INDICE DE VIVACIDAD DE LAS PROVINCIAS}

El índice de vivacidad constituye una medida de la situación de las Provincias españolas en 1974, en cuanto a sus posibilidades inmediatas de avance en su desarrollo, y señala en qué dirección se aparta cada Provincia de la marcha normal y, con su valor, la distancia. Su cálculo se apoya en el índice segundo de capacidad de compra por habitante y su porcentaje medio de variación en 19651974 en cada Provincia. Los valores obtenidos han sido:

\begin{tabular}{|c|c|c|c|}
\hline Provincias & $\begin{array}{l}\text { Indice de } \\
\text { vivacidad }\end{array}$ & Provincias & $\begin{array}{l}\text { Indice de } \\
\text { vivacidad }\end{array}$ \\
\hline $\begin{array}{lllllll}\text { Alava } & \ldots & \ldots & \ldots & \ldots & \ldots & \ldots\end{array}$ & $-3,5$ & $\begin{array}{llllll}\text { Córdoba } & \ldots & \ldots & \ldots & \ldots & \ldots\end{array}$ & $-5,7$ \\
\hline $\begin{array}{llllll}\text { Albacete } & \ldots & \ldots & \ldots & \ldots & \ldots\end{array}$ & $+15,0$ & Coruña, La $\ldots \begin{array}{llll}\ldots & \ldots & \ldots\end{array}$ & $+22,6$ \\
\hline $\begin{array}{llllll}\text { Alicante } & \ldots & \ldots & \ldots & \ldots & \ldots\end{array}$ & $-12,6$ & $\begin{array}{llllllll}\text { Cuenca } & \ldots & \ldots & \ldots & \ldots & \ldots & \ldots\end{array}$ & $-36,2$ \\
\hline Almería $\ldots \ldots \ldots \ldots \ldots$ & $-1,9$ & $\begin{array}{lllllll}\text { Gerona } & \ldots & \ldots & \ldots & \ldots & \ldots & \ldots\end{array}$ & $+16,4$ \\
\hline 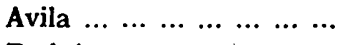 & $+16,6$ & $\begin{array}{llllll}\text { Granada } & \ldots & \ldots & \ldots & \ldots & \ldots\end{array}$ & $-16,1$ \\
\hline Badajoz $\ldots \ldots \ldots \ldots \ldots$ & $-6,6$ & $\begin{array}{lllll}\text { Guadalajara } & \ldots & \ldots & \ldots & \ldots\end{array}$ & $+36,2$ \\
\hline $\begin{array}{llllll}\text { Baleares } & \ldots & \ldots & \ldots & \ldots & \ldots\end{array}$ & $-20,2$ & Guipúzcoa $\ldots \ldots \ldots \ldots$ & $+1,1$ \\
\hline Barcelona $\ldots \ldots \ldots \ldots$ & $-17,0$ & Huelva $\ldots \ldots \ldots \ldots$ & $+18,0$ \\
\hline $\begin{array}{lllllll}\text { Burgos } & \ldots & \ldots & \ldots & \ldots & \ldots & \ldots\end{array}$ & $+11,1$ & $\begin{array}{lllllll}\text { Huesca } & \ldots & \ldots & \ldots & \ldots & \ldots & \ldots\end{array}$ & $+12,1$ \\
\hline Cáceres $\ldots \ldots \ldots \ldots \ldots$ & $-20,5$ & $\begin{array}{lllllll}\text { Jaén } & \ldots & \ldots & \ldots & \ldots & \ldots & \ldots\end{array}$ & $+3,9$ \\
\hline $\begin{array}{lllllll}\text { Cádiz } & \ldots & \ldots & \ldots & \ldots & \ldots & \ldots\end{array}$ & $+1,2$ & $\begin{array}{lllllll}\text { León } & \ldots & \ldots & \ldots & \ldots & \ldots & \ldots\end{array}$ & $+1,2$ \\
\hline $\begin{array}{lllllll}\text { Castellón } & \ldots & \ldots & \ldots & \ldots & \ldots\end{array}$ & $+18,6$ & $\begin{array}{lllllll}\text { Lérida } & \ldots & \ldots & \ldots & \ldots & \ldots & \ldots\end{array}$ & $-20,5$ \\
\hline Ciudad Real $\ldots$... & $-14,3$ & Logroño $\ldots \ldots \ldots \ldots \ldots$ & $+17,5$ \\
\hline
\end{tabular}




\begin{tabular}{|c|c|c|c|}
\hline Provincias & $\begin{array}{l}\text { Indice de } \\
\text { vivacidad }\end{array}$ & Provincias & $\begin{array}{l}\text { Indice de } \\
\text { vivacidad }\end{array}$ \\
\hline Lugo $\ldots \ldots \ldots \ldots \ldots$ & $+3,5$ & Santander $\ldots \ldots \ldots \ldots$ & $+3,6$ \\
\hline 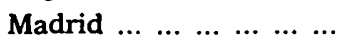 & $+22,1$ & $\begin{array}{llllll}\text { Segovia } & \ldots & \ldots & \ldots & \ldots & \ldots\end{array}$ & $-5,0$ \\
\hline $\begin{array}{lllllll}\text { Málaga } & \ldots & \ldots & \ldots & \ldots & \ldots & \ldots\end{array}$ & $-25,1$ & $\begin{array}{llllllll}\text { Sevilla } & \ldots & \ldots & \ldots & \ldots & \ldots & \ldots\end{array}$ & $-6,4$ \\
\hline $\begin{array}{lllllll}\text { Murcia } & \ldots & \ldots & \ldots & \ldots & \ldots & \ldots\end{array}$ & $+6,5$ & $\begin{array}{lllllll}\text { Soria } & \ldots & \ldots & \ldots & \ldots & \ldots & \ldots\end{array}$ & $-22,2$ \\
\hline $\begin{array}{llllll}\text { Navarra } & \ldots & \ldots & \ldots & \ldots & \ldots\end{array}$ & $-6,0$ & $\begin{array}{llllll}\text { Tarragona } & \ldots & \ldots & \ldots & \ldots & \ldots\end{array}$ & $+25,9$ \\
\hline $\begin{array}{lllllll}\text { Orense } & \ldots & \ldots & \ldots & \ldots & \ldots & \ldots\end{array}$ & $+18,1$ & 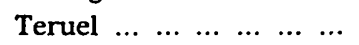 & $+14,9$ \\
\hline $\begin{array}{lllllll}\text { Oviedo } & \ldots & \ldots & \ldots & \ldots & \ldots & \ldots\end{array}$ & $-5,1$ & $\begin{array}{llllllll}\text { Toledo } & \ldots & \ldots & \ldots & \ldots & \ldots & \ldots\end{array}$ & $-0,6$ \\
\hline Palencia $\ldots \ldots \ldots \ldots \ldots$ & $+2,4$ & $\begin{array}{llllll}\text { Valencia } & \ldots & \ldots & \ldots & \ldots & \ldots\end{array}$ & $+1,5$ \\
\hline Palmas, Las $\ldots \ldots \ldots c c$ & $-30,5$ & Valladolid $\ldots \ldots \ldots \ldots$ & $+1,1$ \\
\hline Pontevedra $\ldots \ldots \ldots \ldots$ & $+14,0$ & $\begin{array}{lllllll}\text { Vizcaya } & \ldots & \ldots & \ldots & \ldots & \ldots & \ldots\end{array}$ & $+13,5$ \\
\hline $\begin{array}{llllll}\text { Salamanca } & \ldots & \ldots & \ldots & \ldots & \ldots\end{array}$ & $-17,6$ & Zamora $\ldots \ldots \ldots \ldots$ & $+1,0$ \\
\hline Santa Cruz de Tenerife. & $-14,1$ & $\begin{array}{cccccc}\text { Zaragoza } & \ldots & \ldots & \ldots & \ldots & \ldots\end{array}$ & $-8,0$ \\
\hline
\end{tabular}

Con valores positivos, aunque de distinto nivel, aparecen 27 Provincias, entre las que destacan Guadalajara, demográficamente empobrecida, Tarragona, La Coruña, en distinto grado de industrialización, y Madrid, que constituye, además del centro administrativo del país, una zona industrial y de servicios de primer orden.

En conjunto, aparecen Galicia, seis Provincias del Valle del Duero y dos de la Submeseta Sur; Santander, Vizcaya y Guipúzcoa; tres del Valle del Ebro; cinco del litoral mediterráneo, y tres andaluzas, llamadas a un arranque hacia el desarrollo o su incremento a niveles superiores a los que han conseguido hasta el momento.

\section{LA EVOLUCION DEL CONJUNTO DE MUNICIPIOS ENTRE 1.000 Y 3.000 HABITANTES}

Con periodicidad bienal ha presentado el Anuario los resultados de las investigaciones estadísticas repetidas con referencia a los Municipios de población comprendida entre los 1.000 y 3.000 habitantes, que en 1974 son 1.898, con una extensión superficial de $128.807 \mathrm{~km}^{2}$, el 25,5 por 100 de la superficie del país, y 3,3 millones de habitantes, el 9,7 por 100 de la población total.

En la edición que comentamos presenta un cuadro numérico en el que se sintetiza la evolución en el período 1964-1974, referida a los datos básicos utilizados en las citadas investigaciones. Tomamos los datos correspondientes a los años extremos del período, elaborando los porcentajes de incremento registrados. 


\begin{tabular}{|c|c|c|c|}
\hline & 1964 & 1974 & $\begin{array}{l}\text { Porcenta- } \\
\text { jes de } \\
\text { incremento }\end{array}$ \\
\hline $\begin{array}{lllllllllllll}\text { Teléfonos } & \ldots & \ldots & \ldots & \ldots & \ldots & \ldots & \ldots & \ldots & \ldots & \ldots & \ldots & \ldots\end{array}$ & 92.603 & 259.284 & 280,0 \\
\hline $\begin{array}{llllllllllllll}\text { Camiones } & \ldots & \ldots & \ldots & \ldots & \ldots & \ldots & \ldots & \ldots & \ldots & \ldots & \ldots & \ldots\end{array}$ & 17.403 & 60.690 & 348,7 \\
\hline $\begin{array}{lllllllll}\text { Instrumentos notariales } & \ldots & \ldots & \ldots & \ldots & \ldots & \ldots & \ldots\end{array}$ & 43.870 & 75.645 & 172,4 \\
\hline Recaudación impuesto espectáculos. Año 1966. & $13,3(1)$ & $20,8(1)$ & 156,1 \\
\hline Presupuestos municipales $\ldots \ldots \ldots \ldots \ldots$ & $1.280,2(1)$ & $4.831,5(1)$ & 377,5 \\
\hline $\begin{array}{lllllllllll}\text { Oficinas bancarias } & \ldots & \ldots & \ldots & \ldots & \ldots & \ldots & \ldots & \ldots & \ldots\end{array}$ & 150 & 343 & 228,7 \\
\hline Oficinas Cajas de Ahorro $\ldots \ldots \ldots \ldots \ldots$ & 443 & 1.392 & 314,2 \\
\hline
\end{tabular}

(1) Millones de pesetas.

De su examen se deduce el notable progreso operado en estos Municipios durante dicho período.

\section{ANALISIS ESPECIAL DE VALENCIA Y SU CONTORNO}

Como complemento de las encuestas realizadas en 1972 y 1974 , con respecto a los conjuntos urbanos de Madrid y Barcelona, se ha llevado a cabo una nueva encuesta sobre el mercado de Valencia y su contorno, que constituye «una típica conurbación de características bien definidas», habiéndose determinado «el grado de consumo, hábitos de compra y equipamiento de la familia".

El contorno de Valencia, a efectos de la encuesta, está formado por los términos municipales de: Alboraya, Tabernes Blanques, Bonrepós y Mirambell; Burjasot, Godella y Rocafort, al norte de la capital; Paterna, Manises, Cuart de Poblet, Mislata y Chirivella, que la circundan por el Oeste, y Picaña, Benetúser, Sedaví, Masanasa, Alfafar, Catarroja y Albal, que la rodean por el Sur, constituyendo una concentración humana de 877.654 habitantes, según el Censo de 1970, de ellos 653.890 en la capital y 223.964 en los Municipios del entorno. Los índices, base $1920=100$, eran, en $1970,281,8$ para Valencia y su entorno, 260,2 para Valencia capital y 372,4 para el contorno, lo que denota el más rápido ritmo de crecimiento de los Municipios del contorno.

Los resultados de la encuesta, abstracción hecha de las categorías de consumo con que se enriquece la misma, y de las que prescindimos en este comentario por razones de espacio, se resumen a continuación en sus características más sobresalientes: 


\section{Condiciones de la vivienda}

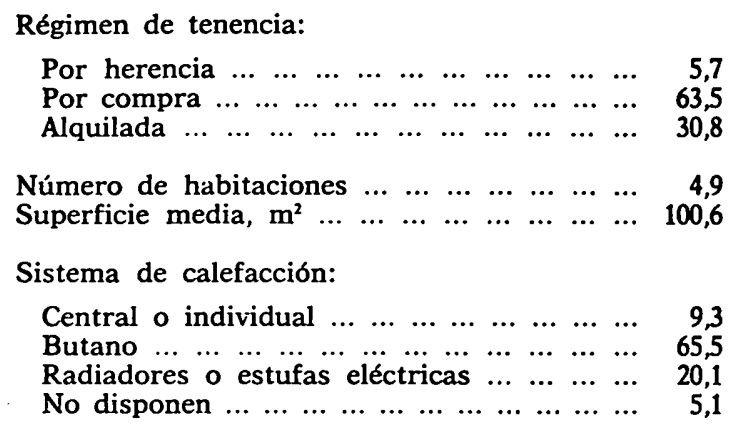

Es de resaltar el alto porcentaje de la vivienda en propiedad, el promedio de habitaciones por vivienda y su superficie media, teteniendo en cuenta que el tamaño medio del hogar es de 3,76 personas. El período de tiempo de uso de la calefacción, debido a las condiciones climáticas, es reducido, $\mathrm{y}$, como se aprecia, los gases licuados y la electricidad son los combustibles más consumidos.

\section{Consumo y hábitos de compra de los hogares}

\begin{tabular}{|c|c|}
\hline \multirow{2}{*}{\multicolumn{2}{|c|}{ 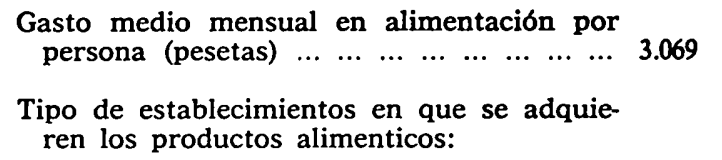 }} \\
\hline & \\
\hline 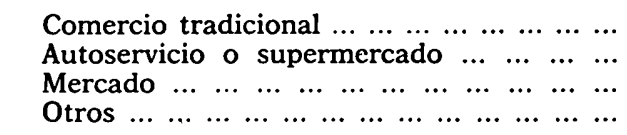 & $\begin{array}{r}20,2 \\
20,1 \\
58,4 \\
1,3\end{array}$ \\
\hline $\begin{array}{l}\text { Tipo de establecimientos en que se adquie- } \\
\text { ren los artículos de vestir: }\end{array}$ & \\
\hline 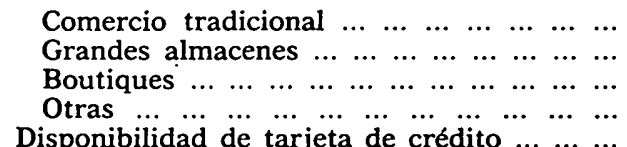 & $\begin{array}{r}34,2 \\
58,7 \\
4,3 \\
2,8 \\
21,2\end{array}$ \\
\hline
\end{tabular}

La adquisición de alimentos se realiza aún, en forma predominante, en mercados, aun cuando se está implantando con éxito el supermercado y el autoservicio. En cuanto a artículos de vestir, ya domina el gran almacén al comercio tradicional. 


\section{3." Equipamiento de los hogares}

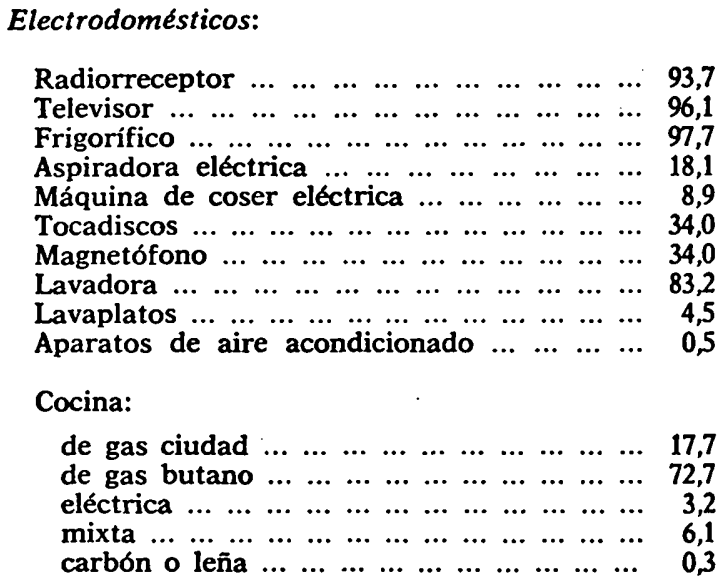

Otros artículos del hogar:

$\begin{array}{lllllllllllllll}\text { Teléfono } & \ldots & \ldots & \ldots & \ldots & \ldots & \ldots & \ldots & \ldots & \ldots & \ldots & \ldots & \ldots & 50,2\end{array}$

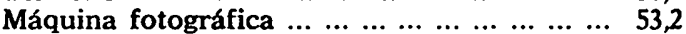

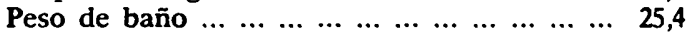

$\begin{array}{lllllllllllllll}\text { Tomavistas } & \ldots & \ldots & \ldots & \ldots & \ldots & \ldots & \ldots & \ldots & \ldots & \ldots & \ldots & \ldots & 7,9\end{array}$

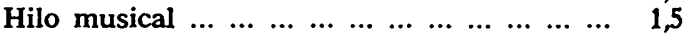

Vehículos:

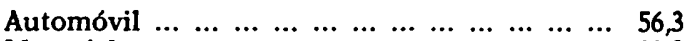

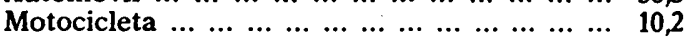

$\begin{array}{lllllllllllllll}\text { Bicicleta } & \ldots & \ldots & \ldots & \ldots & \ldots & \ldots & \ldots & \ldots & \ldots & \ldots & \ldots & \ldots & \ldots & 29,1\end{array}$

El radiorreceptor, el televisor, el frigorífico y la lavadora existe prácticamente en casi todos los hogares, y una tercera parte de ellos poseen tocadiscos y magnetófono. Predomina la cocina de butano y de gas ciudad. La mitad de los hogares disponen de teléfono, máquina fotográfica y automóvil. 


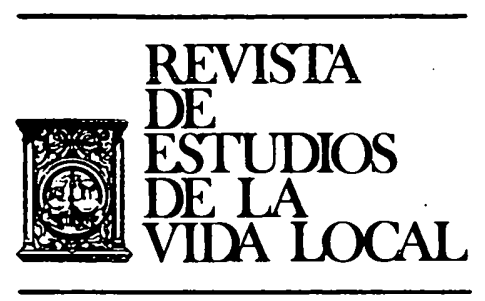

\section{JURISPRUDENCIA}


REVL-1976, núm. 191. BALLESTER ROS, IGNACIO. ALGUNOS ASPECTOS DEL SECTOR DEL COM... 